\title{
Simulation of Detection Effectiveness of Airborne Early Warning Radar
}

\author{
Hong-Tu XIE 1,2,3,a,\#, \\ WANG ${ }^{2}$, Jia-lin SU ${ }^{4}$, Xiao-Tao HUANG ${ }^{3}$, Zhi-Min ZHOU ${ }^{3}$, Guo-Qian WANG ${ }^{5, \#}$ \\ ${ }^{1}$ College of Electrical and Information Engineering, Hunan University, Changsha, China \\ ${ }^{2}$ Air Force Early Warning Academy, Wuhan, China \\ ${ }^{3}$ College of Electronic Science, National University of Defense Technology, Changsha, China \\ ${ }^{4}$ No.63629 Unit, the PLA, Beijing, China \\ ${ }^{5}$ Affiliated Hospital of Hunan Institute of Traditional Chinese Medicine, Changsha, China \\ axht20041623@163.com
}

${ }^{\#}$ Co-first authors and contributed equally to this work. *Corresponding author.

\begin{abstract}
Keywords: Airborne early warning radar, detection effectiveness, simulation, MATLAB
Abstract. Early warning radar is able to effectively detect the target utilizing Doppler Effects between the target and radar. The detection effectiveness of the airborne early warning radar is affected by its detection range as well as its detection probability. In this paper, a method for simulating the detection effectiveness of the airborne early warning radar is proposed. First, the detection model of the airborne early warning radar is designed. And then, based on the motion parameters of the airplane and target as well as the radar system parameter, the detection effectiveness of the early warning radar is simulated and displayed dynamically. Finally, the detection effectiveness of the early warning radar is validated. Simulation results show that the proposed method can effectively simulate the detection effectiveness of the airborne early warning radar, and has the friendly man-machine conversation.
\end{abstract}

\section{Introduction}

Currently, a number of the geosciences and remote sensing applications are developed on the radar system [1]-[4]. In recent years, many early warning radar systems were built by several countries in both military and civilian fields, and then some excellent experiment results were obtained [5]-[7].

Pulse Doppler radar can detect the target using the Doppler Effect [8], and thus it can detect the information of the weak target in the strong land/sea clutter background. Therefore, the pulse Doppler system is widely in the modern early warning radar and airborne fire control radar [9]. As the airborne early warning radar is working, the target located in the radar range maybe not detected, which is caused by the Doppler blind area of the radar [10]. Because as the Doppler velocity of the target is too small, the target is in the Doppler blind area. Therefore, it is necessary to evaluate and simulate the detection effectiveness of the airborne early warning radar before its actual applications.

In [11], the approximate ground/sea clutter model is established based on the analysis of ground/sea clutter characteristics, detection performance of the airborne early warning radar is analyzed adopting classical airborne PD radar signal processing method under the premise of considering mask effect of the fuselage. In [12], by simulating the process of the IRST towing the radar for the detection, a mathematic model for the successful towing probability based on the multi-beam position searching is set up, so searching areas of the radar can be made sure under the condition of the specifically successful towing probability, as well as the expression for the efficiency probability of the radar's detection is acquired from the characteristic of targets. The pattern can hugely reduce the searching areas and time of the radar, and play down time of capturing targets, and then detective probability and detective efficiency of the radar can be improved by using the increase of beam residence time. Based on advantages of the cooperative work of the early warning radar and electronic reconnaissance, the influence of electronic reconnaissance to guide radar search on the detection performance is analyzed [13]. Simulation results show that the heading error of the 
interceptor is increased with the decrease of the intercept, and the distance of the steering probability is decreased when the entering corner is given. A method of evaluating the detection effectiveness is given based on probability analysis method [14], and then the probability distribution function of the detection effectiveness is obtained based on the detection probability density function. Thereby, mapping relationship between the detection range, the detection probability and the detection effectiveness is established.

The purpose of this paper is to design the radar detection model based on the basic information of the carrier aircraft and the target, and then simulates, calculates and dynamic displays the radar detection performance with respect to the target, finally effectively predicts the Doppler velocity blind zone and near-field blind area. According to simulation results, we can arrange flight path in reason or adjust the stance and air route of the carrier aircraft in the actual flight.

\section{Detection Model}

Doppler frequency varies with the relative motion between the airplane and target, and is limited by factors such as the pulse repetition frequency, thus the Doppler frequency of the target can be detected limitedly. There still exists the detection blind area in the radar range of airborne early warning radar.

Fig.1 gives the motion model of airborne early warning radar, protection target and enemy target. The protection target is located in an area, which is determined by relative position between this target and airborne early warning radar. Suppose that the airborne early warning radar flies according to a certain routes in the patrol area, the enemy target may fly from all direction $\left(360^{\circ}\right)$ to the protection target. Based on patrol routes of the airborne early warning radar, according to the radar performance and target characteristics, the detection effectiveness of the airborne early warning radar focusing on the surrounding of the protection area can be analyzed and calculated, i.e., calculating the detection probability of the enemy target from all direction $\left(360^{\circ}\right)$ to the protection area. The established motion model can provide the theoretical basis to find and track enemy target for the early warning radar.

Fig. 2 gives the relative position and velocity between the enemy target and early warning radar. Assuming that the early warning radar is located in the origin of the coordinate system, $\mathrm{M}$ is the position of the enemy target. $r$ is the relative distance between the enemy target and early warning radar, and $r_{x y}$ is the projection of the distance $r$ in the X-Y plane. Similarly, $r_{x}, r_{y}$ and $r_{z}$ are the projections of the distance $r$ in the $\mathrm{X}, \mathrm{Y}$ and $\mathrm{Z}$ axes, respectively. $\alpha, \beta$ and $\gamma$ are the relative angles. $\mathrm{V}_{\mathrm{t}}$ is the sum of velocity vectors of the enemy target and early warning radar. $V_{\text {txy }}$ is the projection of the velocity vector $\mathrm{V}_{t}$ in the $\mathrm{X}-\mathrm{Y}$ plane, and $\mathrm{V}_{\mathrm{tz}}$ is the projection of the velocity vector $\mathrm{V}_{\mathrm{t}}$ in the $\mathrm{Z}$ axis. Thus, the radial velocity of the enemy target with respect to the early warning radar can be considered as the sum of the projection of the velocity vector $\mathrm{V}_{\text {txy }}$ along the $\mathrm{r}$ direction and the projection of the velocity vector $\mathrm{V}_{\mathrm{tz}}$ along the $\mathrm{r}$ direction, which can be calculated by

$$
V_{r}=V_{t x y} \sin \gamma+V_{t z} \cos \gamma
$$

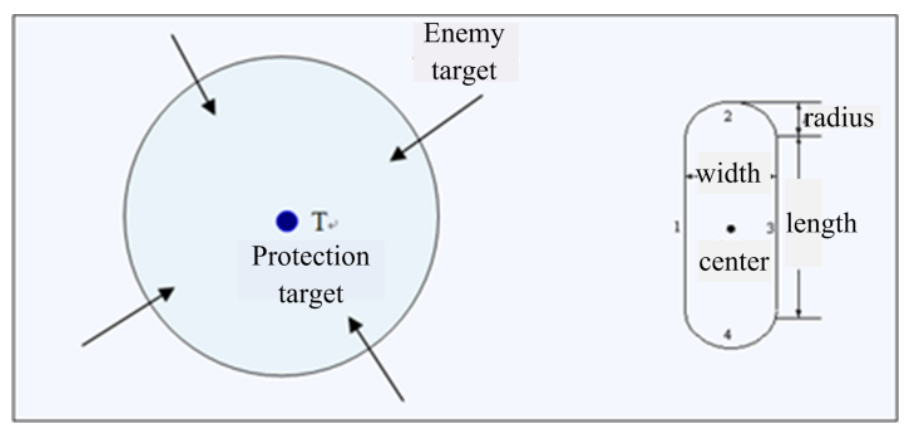

Fig.1 Motion model.

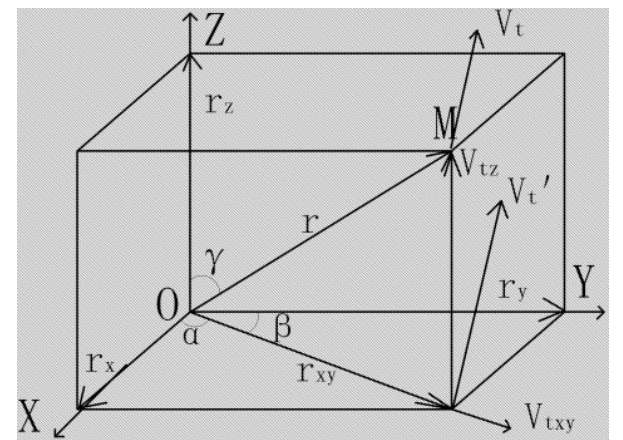

Fig. 2 Relative position and velocity between the enemy target and early warning radar. 
Fig.3 gives the Doppler frequency shift calculation. Assume that all enemy targets within a certain area fly to the center of the area, and the altitudes of the early warning radar and enemy target are constant. The distance between the enemy target and early warning radar is $a, b$ is the distance from the enemy target to regional center, and the distance between the regional center and the early warning radar is c. Thus, the cosine of the angle $\alpha$ can be calculated by

$$
\cos \alpha=\left(a^{2}+b^{2}-c^{2}\right) / 2 a b
$$

Thus, the Doppler frequency shift of the enemy target with respect with the early warning radar is

$$
f_{d}=2 v_{\mathrm{T}} \cos \alpha / \lambda=2 v_{\mathrm{T}}\left(a^{2}+b^{2}-c^{2}\right) /(2 a b \cdot \lambda)
$$

$\mathrm{V}_{\mathrm{T}}$ is the relative velocity of the enemy target with respect to early warning radar, $\lambda$ is the wave length.

Radar's detection probability is related to the false alarm probability, signal-to-noise ratio and radar performance parameters. In engineering applications, calculation of the radar's detection probability is usually calculated using the look-up table method, which is very inconvenient. In this paper, the radar detection model is established based on the Albersheim experience, and then the relationship between the detection probability, false alarm probability and signal-to-noise ratio is given by

$$
S / N=(A+0.12 A B+1.7 B)
$$

$\mathrm{S} / \mathrm{N}$ is the signal-to-noise ratio for pulse accumulation, and

$$
\begin{gathered}
A=\ln \left(0.62 / P_{f a}\right) \\
B=\ln \left[P_{d} /\left(1-P_{d}\right)\right]
\end{gathered}
$$

Where, $\mathrm{P}_{\mathrm{fa}}$ is the false alarm probability and $\mathrm{P}_{\mathrm{d}}$ is the detection probability. When $\mathrm{P}_{\mathrm{fa}}$ is in the range $\left[10^{-7}, 10^{-3}\right]$ and $P_{d}$ is in the range $[0.1,0.9]$, the precision of the Eq. 4 can be accurate to within $0.2 \mathrm{~dB}$.

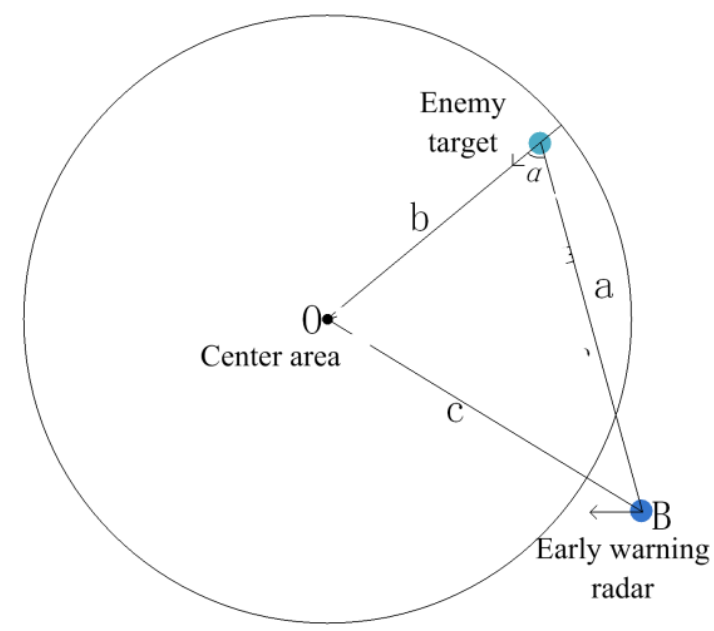

Fig.3 Doppler frequency shift calculation

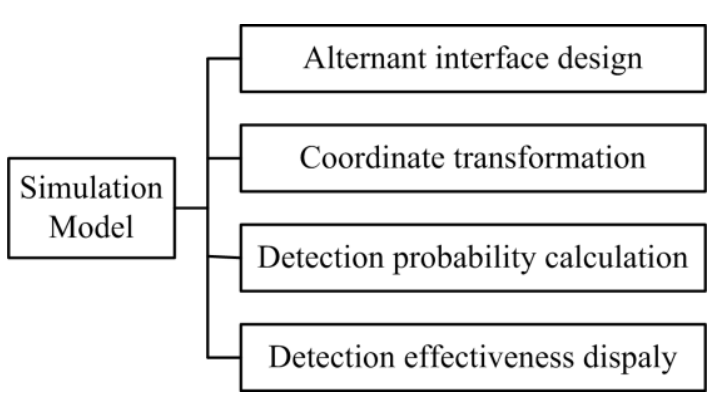

Fig.4 Structure of the simulation model 


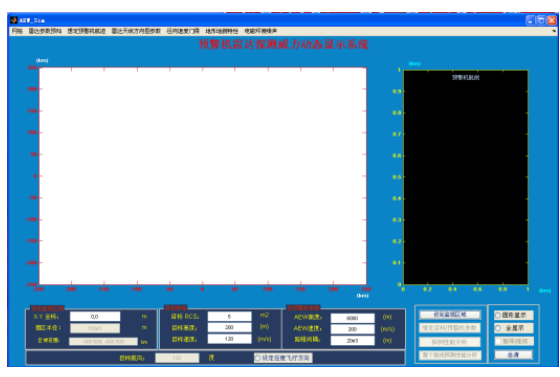

(a)

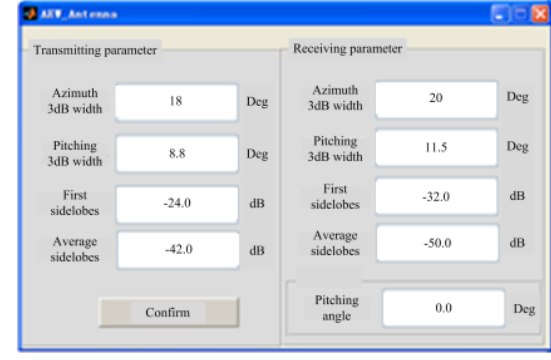

(b)

Fig.5 Alternant interface design. (a) Initialization of the interface; (b) Configuration of the basic parameters.

\section{Design and Implementation}

Fig. 4 shows the structure of the simulation model, which includes the alternant interface design, coordinate transformation, detection probability calculation and detection effectiveness display.

\section{Alternant Interface Design}

MATLAB is usually used to analyze the data, solve the problem and draw the results, thus the graphical user interface (GUI) is not very important. But the friendly interface can help users quickly understand and conveniently operate. However, MATLAB cannot provide the rich function of the graphical interface compared with the other software such as the Visual $\mathrm{C}++$. Thus, it is difficult to provide the concise and friendly GUI using the existing function of the MATLAB graphical interface. The initialization of the interface mainly complete two functions: the initialization of the coordinate system and configuration of the basic parameters, which is shown in Fig.5.

\section{Coordinate Transformation}

It is known that the enemy target and early warning radar are located in the same rectangular coordinate system, but it cannot directly reflect the relative position between the enemy target and early warning radar. Therefore, it is necessary to transform their positions to the body coordinate system. Since the change of the course of the early warning radar will cause the rotating of the body coordinate system, the angle of the enemy target into the radar will change, thus the deviation angle of the early warning radar should be considered. The coordinate transformation is given by

$$
\begin{gathered}
{\left[\begin{array}{l}
X_{D} \\
Y_{D} \\
Z_{D}
\end{array}\right]=\left[\begin{array}{l}
X_{0} \\
Y_{0} \\
Z_{0}
\end{array}\right]+(1+\Delta m) \varepsilon_{x} \varepsilon_{y} \varepsilon_{z}\left[\begin{array}{l}
X \\
Y \\
Z
\end{array}\right]} \\
\varepsilon_{x}=\left[\begin{array}{ccc}
1 & 0 & 0 \\
0 & \cos \varepsilon_{x} & \sin \varepsilon_{x} \\
0 & -\sin \varepsilon_{x} & \cos \varepsilon_{x}
\end{array}\right], \quad \varepsilon_{y}=\left[\begin{array}{ccc}
\cos \varepsilon_{y} & 0 & -\sin \varepsilon_{y} \\
0 & 1 & 0 \\
\sin \varepsilon_{y} & 0 & \cos \varepsilon_{y}
\end{array}\right], \quad \varepsilon_{z}=\left[\begin{array}{ccc}
\cos \varepsilon_{z} & \sin \varepsilon_{z} & 0 \\
-\sin \varepsilon_{z} & \cos \varepsilon_{z} & 0 \\
0 & 0 & 1
\end{array}\right]
\end{gathered}
$$

$\left(X_{0}, Y_{0}, Z_{0}\right)$ is the value of the origin of the original coordinate system in the new coordinate system, $(X, Y, Z)$ is the value of the enemy target in the original coordinate system, and $\left(X_{\mathrm{D}}, Y_{\mathrm{D}}, Z_{\mathrm{D}}\right)$ is the value of the enemy target in the new coordinate system. $\varepsilon_{x}, \varepsilon_{y}, \varepsilon_{z}$ are the rotation parameters between the original coordinate system and specific coordinate axes. $\Delta \mathrm{m}$ is the scale parameters between these two coordinate systems. Based on the known parameters, the relative position between the enemy target and early warning radar can be calculated at any time.

\section{Detection Probability Calculation}

The idea of calculating the detection probability of the enemy target is as follows:

a) Giving the radar system detection performance, $P_{d}=0.8, P_{f a}=10^{-6}$, sigma $=1 \mathrm{~m}^{2}, R_{\max }=400 \mathrm{~km}$. 
b) Using the Albersheim formula to calculate the signal-to-noise ratio $\mathrm{S} / \mathrm{N}$ when $\mathrm{P}_{\mathrm{d}}=0.8$ and $\mathrm{P}_{\mathrm{fa}}=$ $10^{-6}$, which is regarded as the reference value for calculating detection ability of the monitoring area.

c) Based on given parameters of the enemy target (the radar cross section, speed and coordinates), the corresponding signal-to-noise ratio $\mathrm{S} / \mathrm{N}$ can be calculated according to the steps a) and $\mathrm{b}$ ).

d) According to the signal-to-noise ratio $\mathrm{S} / \mathrm{N}$, Albersheim formula and Doppler threshold, the detection ability of the enemy target can be calculated, which can show the detection effectiveness.

In the real design and application of the early warning radar, the high detection probability and low false-alarm probability should be simultaneity needed. To solve this contradiction, the constant false alarm technology is usually used, which can maintain a constant low false alarm probability and obtain the higher detection probability. Fig. 6 gives the relationship between the detection probability and distance of the enemy target, where the false-alarm probability is equal to $10^{-6}$. From Fig.6, it can be found that the detection probability of the case $(m=3)$ is higher than that of the case $(m=1)$.

\section{Detection Probability Display}

After the route configuration of the early warning radar and enemy target, movement information of the early warning radar and enemy target is input, as well as the Doppler velocity threshold, the detection probability of enemy targets can be dynamically display. Fig.7 gives the dynamic simulation results of the detection probability of the early warning radar. Fig. 8 shows the graphical user home interface of the simulation model. Simulation parameters of the dynamic display are given as follows:

a) The real-time position of the early warning radar is represented by the sign " $2 \gamma^{\prime}$ ".

b) The enemy target: (1) red represents the area of the high detection probability, i.e., $P_{d}>0.8$; (2) yellow represents the area of the valid detection probability, i.e., $P_{d}$ between 0.3 and 0.8 ; (3) blue represents that the enemy target is in radar blind area or outside the detection range, i.e., $\mathrm{P}_{\mathrm{d}}<0.3$.

c) The early warning radar: (1) red " $O$ " is on behalf of the sample point position for the radar; (2) the white line is on behalf of the radar flight path.

After the dynamic display, the model can also provide the total information such as the detection probability of the sampling points, each point.

\section{Summary}

In this paper, a new method for simulating the detection effectiveness of the airborne early warning radar is proposed. First, the detection model of the airborne early warning radar is designed. And then, based on the motion parameters of the airplane and target as well as the radar system parameter, the detection effectiveness of the early warning radar is simulated and displayed dynamically. Finally, the detection effectiveness of the early warning radar is tested. Simulation results show that the proposed method is able to effectively simulate the detection effectiveness of the early warning radar, and has friendly man-machine conversation. The proposed method provides some references to the radar tactical index, radar power assessment, and radar operational effectiveness assessment.

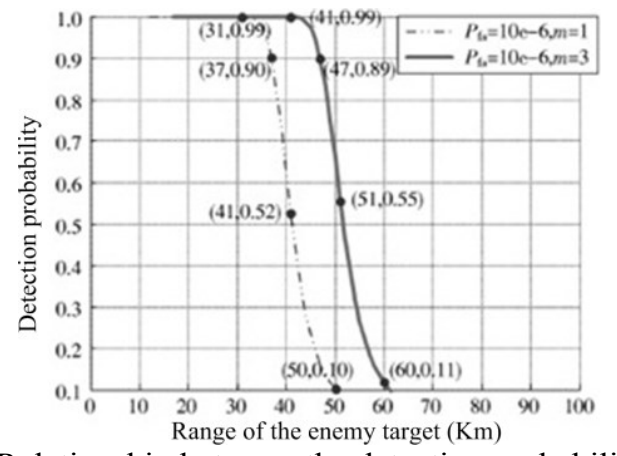

Fig.6 Relationship between the detection probability and distance of the enemy target.

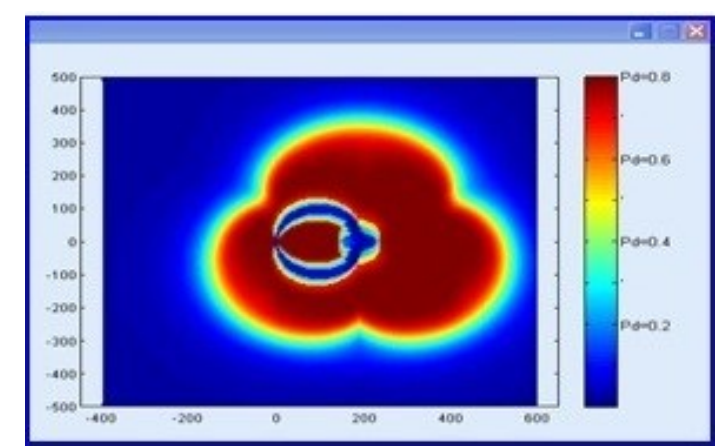

Fig.7 Dynamic simulation results of the detection probability of the early warning radar 


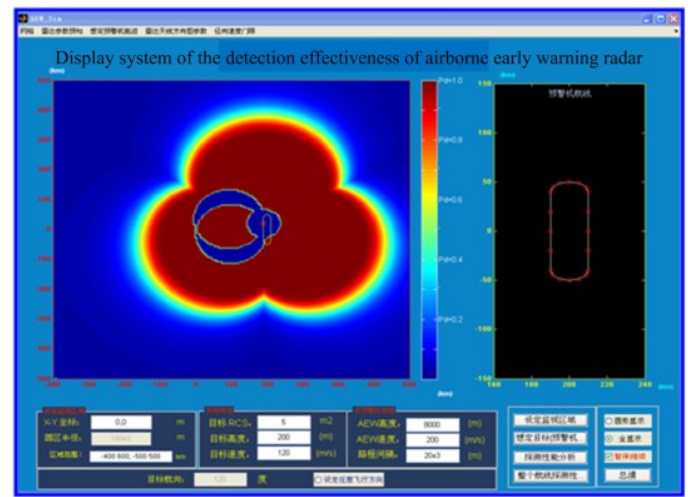

Fig. 8 Graphical user home interface of the simulation model

\section{Acknowledgement}

This work was supported in part by the National Natural Science Foundation of China under Grants 61571447, in part by the Scientific Research Project of Education Department of Hunan under Grant 16C1233, and in part by the Domain Foundation of Equipment Advance Research of 13th Five-year Plan under Grants 6140410301 and 6140410304. Hongtu Xie and Guoqian Wang are the co-first authors and contributed equally to this work, and Shaoying Shi is the corresponding author.

\section{References}

[1] D. An, Y. Li, X. Huang, X. Li, and Z. Zhou, "Performance evaluation of frequency-domain algorithms for chirped low frequency UWB SAR data processing," IEEE J. Sel. Topics Appl. Earth Observ., vol. 7, no. 2, pp. 678-690, 2014.

[2] H. Xie, D. An, X. Huang, and Z. Zhou, "Efficient raw signal generation based on equivalent scatterer and subaperture processing for one-stationary bistatic SAR including motion errors," IEEE Trans. Geosci. Remote Sens., vol.54, no.6, pp.3360-3377, 2016.

[3] H. Xie, D. An, X. Huang, and Z. Zhou, "Research on spatial resolution of one-stationary bistatic ultrahigh frequency ultrawidebeam-ultrawideband SAR," IEEE J. Sel. Topics Appl. Earth Observ., vol. 8, no. 4, pp. 1782-1798, 2015.

[4] H. Xie et al, "Fast factorized backprojection algorithm for one-stationary bistatic spotlight circular SAR image formation," IEEE J. Sel. Topics Appl. Earth Observ., vol. 10, no. 4, pp. 1494-1510, 2017.

[5] L. Zhang and Y. Xu, "Prospect for technology of airborne early warning radar", Modern Radar, vol.37, no.1, pp.1-7, 2015.

[6] C. Cao, "Development of AWACS for 70 years", Journal of China Academy of Electronics and Information Technology, vol.10, no.4, pp.1-6, 2015.

[7] J. Lu et al. Introduction to Airborne Early Warning System. 2nd ed. Beijing, China: National Defense Industry Press, 2011.

[8] X. Lian, "MPRF design and selection of airborne PD radar", Modern Defence Technology, vol.44, no.4, pp.129-135, 2016.

[9] Y. Huang, and C. Ding, "Track deception jamming and auxiliary decision making research of airborne early warning PD radar", Modern Radar, vol.37, no.11, pp. 77-80, 2015.

[10]X. Wang et al, "Flight planning of low-altitude penetration based on Doppler blind zone", Systems Engineering and Electronics, vol.37, no.7, pp. 1531-1535, 2015. 
[11]X. Lian, Z. Zhu, X. Wang, F. Yu, and Z. Lei, "Detection performance analysis of AEW radar range under complex clutter background", Shipboard Electronic Countermeasure, vol.38, no.5, pp. 19-24, 2015.

[12]F. Peng, J. Wu, J. Xiang, W. Long, and J. Ma, "Evaluation and simulation of detection efficiency based on using IRST sensor towing the airborne early warning radar", Modern Radar, vol.38, no.1, pp. $5-9,2016$.

[13] J. Ma, F. Peng, and L. Wu, "Influence of cooperative work of the airborne early warning radar and ESM on the combat effectiveness", Journal of Xi'an Technological University, vol.35, no.10, pp. 843-849, 2015.

[14]J. Ma, and Y. Fan, "Study of airborne early warning radar's detection effectiveness based on probability analysis", Journal of Air Force Engineering University, vol.16, no.5, pp. 1-4, 2015. 